\title{
Pharmacokinetically-guided dosing to improve the efficacy of brigatinib in non-small cell lung cancer patients
}

\author{
Simon Koele ${ }^{1}$, Stijn van Beek ${ }^{2}$, Anthonie van der Wekken ${ }^{3}$, Berber Piet ${ }^{4}$, Michel van den \\ Heuvel $^{4}$, and Rob ter Heine ${ }^{1}$ \\ ${ }^{1}$ Radboud University Medical Center, Radboud Institute for Health sciences \\ ${ }^{2}$ Radboud University Medical Cente, Radboud Institute for Health Sciences \\ ${ }^{3}$ University of Groningen, University Medical Centre Groningen \\ ${ }^{4}$ Radboud University Medical Center
}

July 7,2021

\begin{abstract}
Brigatinib was recently approved for the treatment of anaplastic lymphoma kinase-positive non-small cell lung cancer and is dosed according to a one-dose-fits-all paradigm. We aimed to identify a pharmacokinetically-guided precision dosing strategy to improve treatment response with brigatinib through simulations using a previously published pharmacokineticpharmacodynamic model. Dosing strategies explored were the approved 180mg QD, the highest tolerable dose tested in clinical trials: 240mg QD, and two precision dosing strategies targeting the median trough concentrations following 180mg QD, and 240mg QD. We investigated the impact of alternative dosing regimens on progression-free survival (PFS), overall survival (OS), and the probability of developing a grade [?]2 rash or grade [?]2 amylase increase. Median PFS and OS increased by 1.6 and 7.8 months, respectively between the currently approved dosing strategy and precision dosing to the median trough concentration of the $240 \mathrm{mg}$ dosing strategy, with only a minor increase in the probability of developing toxicity.
\end{abstract}

\section{Hosted file}

Pharmacokinetically-guided dosing to improve the efficacy of brigatinib in non-small cell lung cancer p available at https://authorea.com/users/424375/articles/529476-pharmacokinetically-guideddosing-to-improve-the-efficacy-of-brigatinib-in-non-small-cell-lung-cancer-patients 